\section{New Coupling Reaction of $o$-Phenylenediamine Catalyzed by Mn(II) Ion}

M. L. HSIEH, M. C. CHENG and S. M. PENG*

Department of Chemistry, National Taiwan University Taipei 10764, Taiwan

(Received October 27, 1987)

As a result of research on metal complexes with benzoquinonediimine (bqdi) and semi-benzoquinonediimine (s-bqdi) radical anion, a lot of work has been reported $[1-6]$. Balch and Holm well characterized the metal complexes $(\mathrm{M}=\mathrm{Co}(\mathrm{II}), \mathrm{Ni}(\mathrm{II})$,

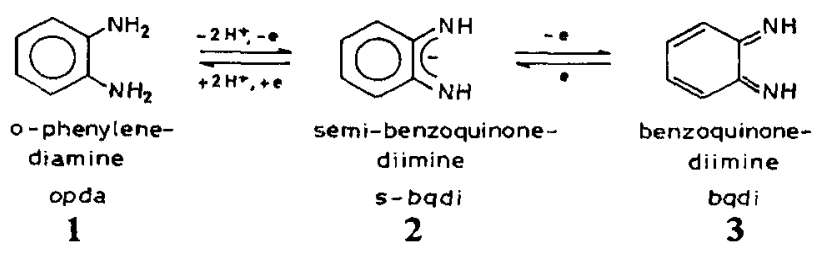

Pd(II), Pt(II)) of 2 [1]. The crystal structure of [Ni(II)(s-bqdi) $\left.)_{2}\right]$ has been reported by Hall and Soderberg [2]. Christoph and Goedken synthesized the $\mathrm{Fe}$ (II) complex of 3 and reported the structure of $\left[\mathrm{Fe}(\mathrm{II})(\mathrm{CN})_{4} \text { (bqdi) }\right]^{2-}$ [3]. A series of interesting st ructures $\left[\mathrm{Fe}(\mathrm{II})\left(\mathrm{bqdi}_{3}\right]\left(\mathrm{PF}_{6}\right)_{2}, \quad\left[\mathrm{Co}(\mathrm{II})(\mathrm{s}-\mathrm{bqdi})_{2}\right]\right.$ and $\left[\mathrm{Co}\right.$ (III)Cl(s-bqdi) $\left.{ }_{2}\right]$ have been determined by us [4]. The dimerized coupling reactions of 1 catalyzed by transition metal ions have also been reported $[5,6]$.

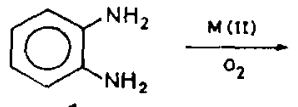

1

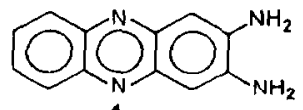

4
We here report the new trimerized coupling reaction of 1 and the crystal structure of [Mn(II)(tribqdi) $\left.)_{2}\right]\left(\mathrm{ClO}_{4}\right)_{2}(5)$.

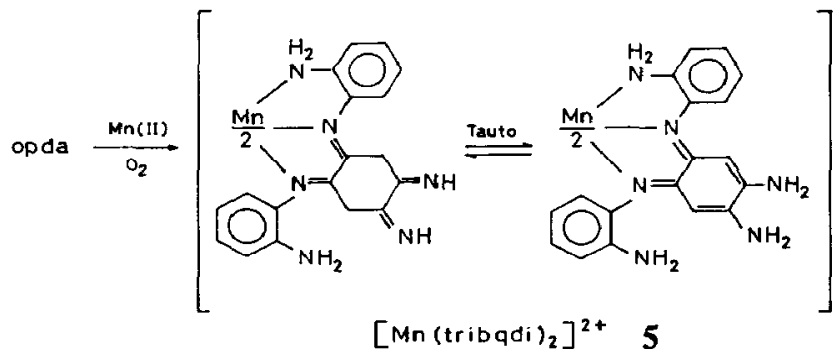

*Author to whom correspondence should be addressed. Also associated with Institute of Chemistry, Academia Sinica, Nankang, Taipei, 11529, Taiwan.

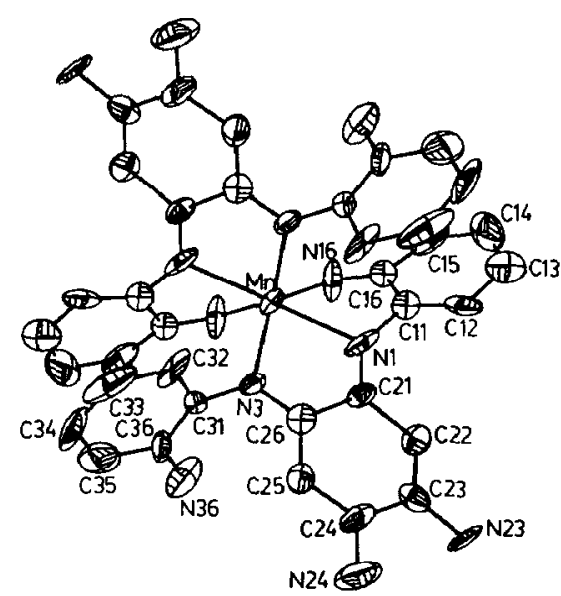

Fig. 1. ORTEP drawing of the cation [Mn(tribqdi) $\left.)_{2}\right]^{2+}$ with $50 \%$ probability. Space group $C 2 / c, a=17.084(14), b=$ 12.238(8), $c=22.142(15) A, \beta=104.13(5)^{\circ}, Z=4,1151$ reflections with $r>2.5 \sigma(I), 253$ variables, $R=11 \%, R_{\mathrm{w}}=$ $11 \%$. Complex ion lies on a crystallographic 2 -fold.

The reaction of $M n(I I)$ perchlorate with opda and $\mathrm{O}_{2}$ in methanol solution for a few hours, followed by precipitation by adding diethyl ether, leads to the isolation of a dark green crystalline compound, 5. Suitable single crystals were obtained by slow diffusion of ether into a methanol solution of 5 .

The result of the X-ray structural analysis of 5 shown in Fig. 1, and tabulated in Table I, confirms the trimerization of the oxidized opda ligand. The central $\mathrm{Mn}$ atom, located at crystallographic 2-fold, has a distorted octahedral coordination. The long $\mathrm{Mn}-\mathrm{N}$ distances, $2.18(1), 2.25(1)$ and $2.34(2) \AA$ indicate the high spin state of the complex and are attributed to the crowded coordination sphere. In the interesting tridentate ligand, the $\mathrm{N} 1-\mathrm{C} 21$ and N3-C26 distances, $1.23(2)$ and 1.33(2) $\AA$ respectively, are short, consistent with their identification as double bonds. The $\mathrm{C} 22-\mathrm{C} 23, \mathrm{C} 23-\mathrm{N} 23$, C24-N24 and $\mathrm{C} 24-\mathrm{C} 25$ distances are intermediate between those expected for the single and double bonds. The bond pattern of ring 2 is consistent with the hybrid of those of tetraimine and diiminedienamine. The rings 1 and 3 are normal aromatic rings.

\section{Supplementary Material}

A table of coordinates and thermal parameters of 5 and a structure factor table of 5 are available from the authors on request.

(C) Elsevier Sequoia/Printed in Switzerland 
TABLE I. Bond Lengths $(A)$ and Bond Angles $\left({ }^{\circ}\right)$ of 5

\begin{tabular}{lllllllll}
\hline Atom 1 & Atom 2 & Length & Atom 1 & Atom 2 & Length & Atom 1 & Atom 2 & Length \\
\hline Mn & N1 & $2.25(1)$ & Mn & N1 & $2.25(1)$ & Mn & N3 & $2.18(1)$ \\
Mn & N3 & $2.18(1)$ & Mn & N16 & $2.34(2)$ & Mn & N16 & $2.34(2)$ \\
C1 & O1 & $1.43(2)$ & Cl & O2 & $1.39(2)$ & C1 & O3 & $1.36(2)$ \\
C1 & O4 & $1.35(2)$ & N1 & C11 & $1.40(3)$ & N1 & C21 & $1.23(2)$ \\
N3 & C26 & $1.33(2)$ & N3 & C31 & $1.40(3)$ & N16 & C16 & $1.41(3)$ \\
N23 & C23 & $1.34(2)$ & N24 & C24 & $1.31(3)$ & N36 & C36 & $1.40(3)$ \\
C11 & C12 & $1.40(3)$ & C11 & C16 & $1.40(3)$ & C12 & C13 & $1.37(3)$ \\
C13 & C14 & $1.42(4)$ & C14 & C15 & $1.40(4)$ & C15 & C16 & $1.34(4)$ \\
C21 & C22 & $1.52(3)$ & C21 & C26 & $1.46(3)$ & C22 & C23 & $1.40(3)$ \\
C23 & C24 & $1.45(3)$ & C24 & C25 & $1.42(3)$ & C25 & C26 & $1.46(3)$ \\
C31 & C32 & $1.44(3)$ & C31 & C36 & $1.35(3)$ & C32 & C33 & $1.40(4)$ \\
C33 & C34 & $1.38(4)$ & C34 & C35 & $1.34(4)$ & C35 & C36 & $1.41(3)$
\end{tabular}

\begin{tabular}{|c|c|c|c|c|c|c|c|}
\hline Atom 1 & Atom 2 & Atom 3 & Angle & Atom 1 & Atom 2 & Atom 3 & Angle \\
\hline N1 & $\mathrm{Mn}$ & N1 & $156.5(7)$ & N1 & $\mathrm{Mn}$ & N3 & $72.9(6)$ \\
\hline N1 & $\mathrm{Mn}$ & N3 & $93.3(6)$ & $\mathrm{N} 1$ & $\mathrm{Mn}$ & N16 & $71.8(6)$ \\
\hline N1 & $\mathrm{Mn}$ & N16 & $128.7(6)$ & $\mathrm{N} 1$ & $\mathrm{Mn}$ & N3 & $93.3(6)$ \\
\hline N1 & Mn & N3 & $72.9(6)$ & N1 & $\mathrm{Mn}$ & N16 & $128.7(6)$ \\
\hline N1 & Mn & N16 & $71.8(6)$ & N3 & $\mathrm{Mn}$ & N3 & $108.8(8)$ \\
\hline N3 & $\mathrm{Mn}$ & N16 & $134.4(6)$ & N3 & $\mathrm{Mn}$ & N16 & $101.0(7)$ \\
\hline N3 & $\mathrm{Mn}$ & N16 & $101.0(7)$ & N3 & $\mathrm{Mn}$ & N16 & $134.4(6)$ \\
\hline N16 & Mn & N16 & $80.0(7)$ & $\mathrm{O} 1$ & $\mathrm{Cl}$ & $\mathrm{O} 2$ & $113(1)$ \\
\hline 01 & $\mathrm{Cl}$ & $\mathrm{O} 3$ & $109(1)$ & 01 & $\mathrm{Cl}$ & 04 & $103(1)$ \\
\hline $\mathrm{O} 2$ & $\mathrm{Cl}$ & O3 & $106(1)$ & $\mathrm{O} 2$ & $\mathrm{Cl}$ & 04 & $113(1)$ \\
\hline o3 & $\mathrm{Cl}$ & O4 & $113(1)$ & Mn & N1 & $\mathrm{C} 11$ & $112(1)$ \\
\hline Mn & $\mathrm{N} 1$ & $\mathrm{C} 21$ & $116(1)$ & $\mathrm{C} 11$ & $\mathrm{~N} 1$ & $\mathrm{C} 21$ & $132(1)$ \\
\hline Mn & N3 & $\mathrm{C} 26$ & $116(1)$ & $\mathbf{M n}$ & N3 & C31 & $127(1)$ \\
\hline $\mathrm{C} 26$ & N3 & C31 & $116(1)$ & $\mathrm{Mn}$ & N16 & C16 & $105(1)$ \\
\hline N1 & $\mathrm{C} 11$ & $\mathrm{C} 12$ & $122(2)$ & N1 & $\mathrm{Cl1}$ & $\mathrm{C} 16$ & $114(2)$ \\
\hline $\mathrm{C} 12$ & C11 & C16 & $123(2)$ & $\mathrm{Cl1}$ & $\mathrm{C} 12$ & $\mathrm{C} 13$ & $120(2)$ \\
\hline $\mathrm{C} 12$ & $\mathrm{C} 13$ & $\mathrm{C} 14$ & $119(2)$ & $\mathrm{Cl3}$ & $\mathrm{Cl} 4$ & $\mathrm{C} 15$ & $118(2)$ \\
\hline C14 & $\mathrm{C} 15$ & C16 & $125(2)$ & N16 & $\mathrm{C} 16$ & $\mathrm{C} 11$ & $120(2)$ \\
\hline N16 & $\mathrm{C} 16$ & $\mathrm{C} 15$ & $124(2)$ & $\mathrm{C} 11$ & $\mathrm{C} 16$ & $\mathrm{C} 15$ & $115(2)$ \\
\hline N1 & $\mathrm{C} 21$ & $\mathrm{C} 22$ & $124(2)$ & N1 & $\mathrm{C} 21$ & $\mathrm{C} 26$ & $118(2)$ \\
\hline $\mathrm{C} 22$ & $\mathrm{C} 21$ & $\mathrm{C} 26$ & $118(2)$ & $\mathrm{C} 21$ & $\mathrm{C} 22$ & $\mathrm{C} 23$ & $119(2)$ \\
\hline $\mathrm{N} 23$ & $\mathrm{C} 23$ & $\mathrm{C} 22$ & $119(2)$ & $\mathrm{N} 23$ & $\mathrm{C} 23$ & $\mathrm{C} 24$ & $119(2)$ \\
\hline $\mathrm{C} 22$ & $\mathrm{C} 23$ & $\mathrm{C} 24$ & $122(2)$ & N24 & $\mathrm{C} 24$ & $\mathrm{C} 23$ & $119(2)$ \\
\hline N24 & $\mathrm{C} 24$ & $\mathrm{C} 25$ & $120(2)$ & $\mathrm{C} 23$ & $\mathrm{C} 24$ & $\mathrm{C} 25$ & $121(2)$ \\
\hline $\mathrm{C} 24$ & $\mathrm{C} 25$ & $\mathrm{C} 26$ & $120(2)$ & N3 & $\mathrm{C} 26$ & $\mathrm{C} 21$ & $116(2)$ \\
\hline N3 & $\mathrm{C} 26$ & $\mathrm{C} 25$ & $125(2)$ & $\mathrm{C} 21$ & $\mathrm{C} 26$ & $\mathrm{C} 25$ & $119(2)$ \\
\hline N3 & $\mathrm{C} 31$ & $\mathrm{C} 32$ & $116(2)$ & N3 & C31 & C36 & $119(2)$ \\
\hline $\mathrm{C} 32$ & $\mathrm{C} 31$ & $\mathrm{C} 36$ & $124(2)$ & C31 & $\mathrm{C} 32$ & C33 & $113(2)$ \\
\hline C32 & $\mathrm{C} 33$ & $\mathrm{C} 34$ & $122(2)$ & $\mathrm{C} 33$ & C34 & C35 & $124(2)$ \\
\hline C34 & $\mathrm{C} 35$ & C36 & $117(2)$ & N36 & C36 & $\mathrm{C} 31$ & $124(2)$ \\
\hline N36 & C36 & C35 & $116(2)$ & C31 & C36 & C35 & $120(2)$ \\
\hline
\end{tabular}

\section{Acknowledgement}

We thank the National Research Councils of the Republic of China for support.

\section{References}

1 A. L. Balch and R. H. Holm, J. Am. Chem. Soc, 88 , 5201 (1966).
2 G. S. Hall and R. H. Soderberg, Inorg. Chem., 7, 2300 (1968).

3 G. G. Christoph and V. L. Goedken, I. Am. Chem. Soc. 95, 3869 (1973).

4 S. M. Peng, C. T. Chen, D. S. Liaw, C. I. Chen and Y. Wang, Inorg. Chim. Acta, 101, L31 (1985).

5 M. G. Miles and J. D. Wilson, Inorg. Chem., 14, 2357 (1975).

6 S. M. Peng and D. S. Liaw, Inorg. Chim. Acta, 113, L11 (1986). 